\title{
Comparison of Methods for Counting Podocyte Number in the Kidney
}

\author{
J.M. Basgen,* and S.B. Nicholas, ${ }^{*}$ \\ *Morphometry \& Stereology Laboratory, Department of Research, Charles Drew University of \\ Medicine and Science, Los Angeles, CA 90059 \\ ${ }^{*}$ Divisions of Nephrology and Endocrinology, Department of Medicine, David Geffen School of \\ Medicine at UCLA, Los Angeles, CA 90095
}

The podocyte is a specialized epithelial cell that is crucial in maintaining the structure and function of the glomerulus within the kidney [1]. Pagtalunan et al. first reported a decrease in podocyte number associated with type 2 diabetes [2]. Since that initial report, many other studies have associated a decrease in podocyte number with several kidney diseases [3,4]. Similar reports have observed decreased podocyte number in rat and mouse models of proteinuric renal diseases [5]. However, there is inconsistency in the number of podocytes reported in these studies, possibly due to the use of different methods for podocyte counting. In this study, we report the results from three methods comparing podocyte number obtained from two of the widely used counting methods against a gold standard, thus identifying the more accurate counting method.

To test the methods, 1-mm blocks from a single mouse kidney were embedded in Poly/Bed ${ }^{\circledR} 812$. Serial $1-\mu \mathrm{m}$ thick sections $(\mathrm{n}=250)$ were cut and podocyte number was counted in nine glomeruli with each of the three methods. Digital images from all sections throughout the nine glomeruli $(\sim 80$ sections/glomerulus) were obtained using the Exhaustive Cut method. This method allowed all podocyte nuclei to be counted as they were sequentially observed, thus avoiding sampling error (Fig. 1) which is considered the gold standard. On the other hand, using the less time-consuming WeibelGomez method the number of podocyte nuclei profiles were counted, and the volume fraction of podocyte nuclei per glomerulus was determined using a counting grid superimposed over the image (Fig. 2). Shape and variance factors were determined and glomerular volume was also measured followed by calculation of podocyte number [6]. For the Fractionator/Disector method, pairs of consecutive sections were used to determine the number of podocytes. The number of profiles from podocytes that were present in the Sampling Section but absent in the Look-up Section (Fig. 3) was used to calculate podocyte number [7].

From the Exhaustive Cut method $84.3 \pm 7.7$ (mean \pm SD) podocytes were counted compared with $89.3 \pm 6.8$ podocytes using the Weibel-Gomez method, which was statistically significantly different, $\mathrm{p}<0.001$. From the Fractionator/Disector method, $81.9 \pm 13.3$ were counted, with no statistical difference compared with the gold standard, $p>0.30$. Therefore, this study suggests that the unbiased Fractionator/Disector method is the recommended approach for counting podocyte number.

References

[1] W. Kriz et al., Kidney Int 45 (1994) 396

[2] M.E. Pagtalunan et al., J Clin Invest 99 (1997) 342

[3] M.W. Steffes et al., Kidney Int 59 (2001) 2104

[4] K.E. White et al., Diabetes 51 (2002) 308

[5] J.M. Teiken et al., Anat Rec 291 (2007) 114

[6] E.R. Weibel and D.M. Gomez, J Appl Physiol 17 (1962) 343

[7] H.J.G. Gundersen, J Microsc 147 (1987) 3 
Fig 1. Exhaustive Cut. First 8 sections from a glomerulus showing the first 4 podocyte nuclei.

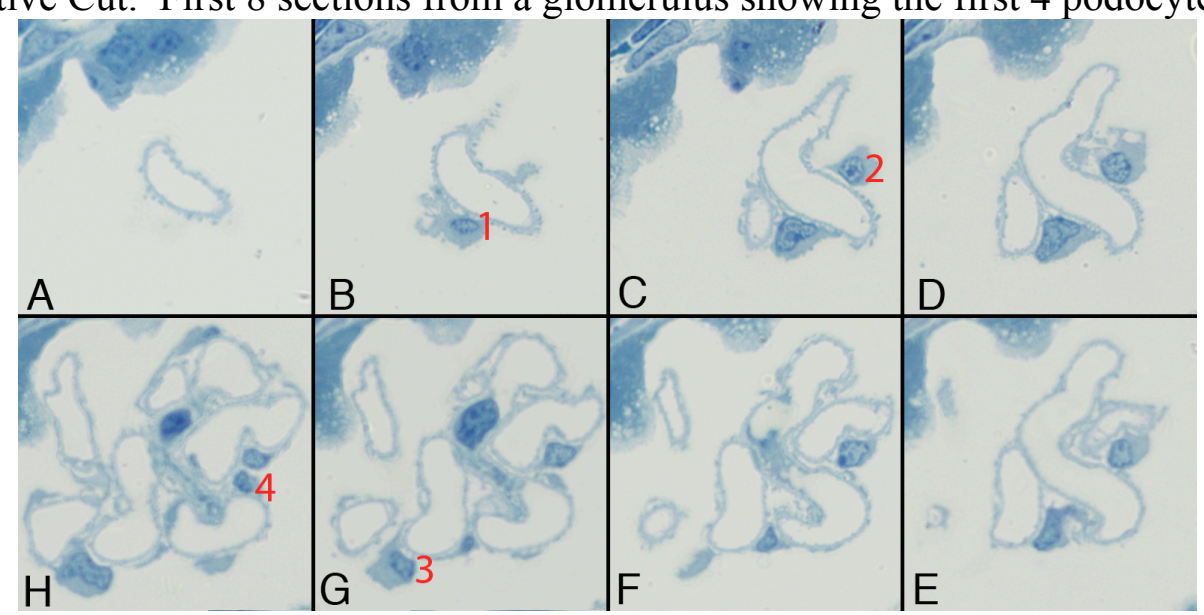

Fig. 2. Weibel-Gomez. Twelve podocyte nuclei profiles counted then a counting grid used to measured fractional volume of podocyte nuclei per glomerulus.

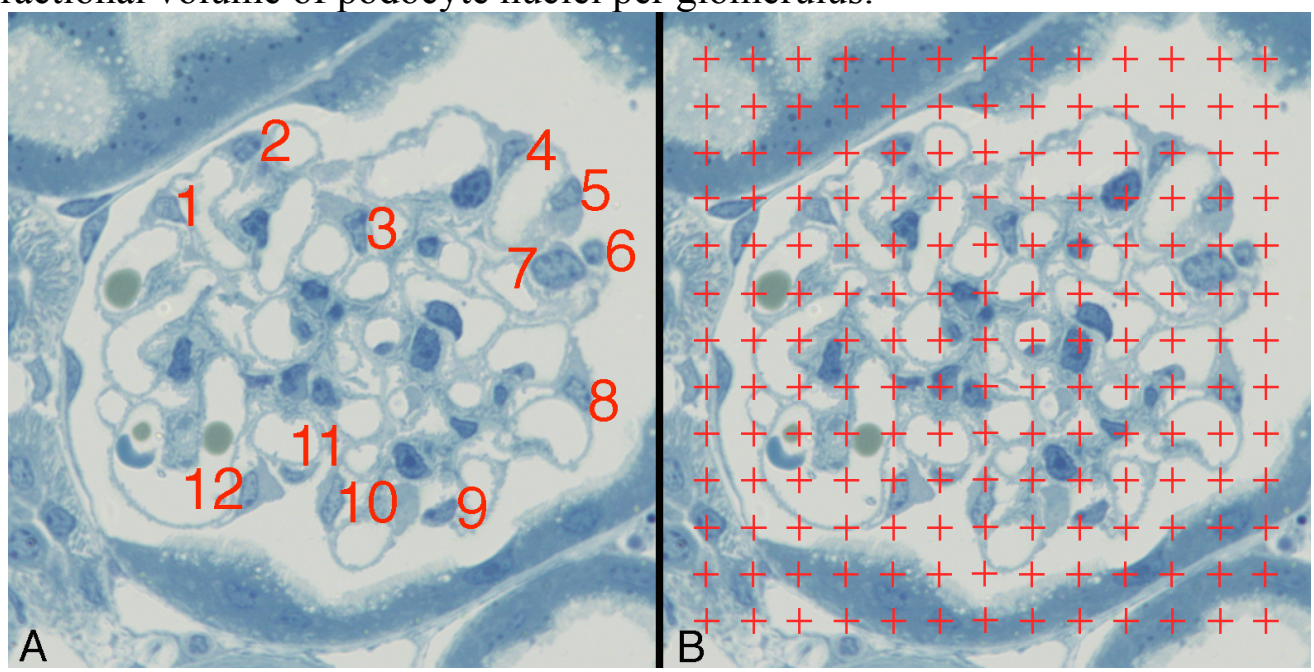

Fig 3. Fractionator/Disector. Profiles are seen from three podocyte nuclei present in Sampling Section that are not present in Look-up Section.

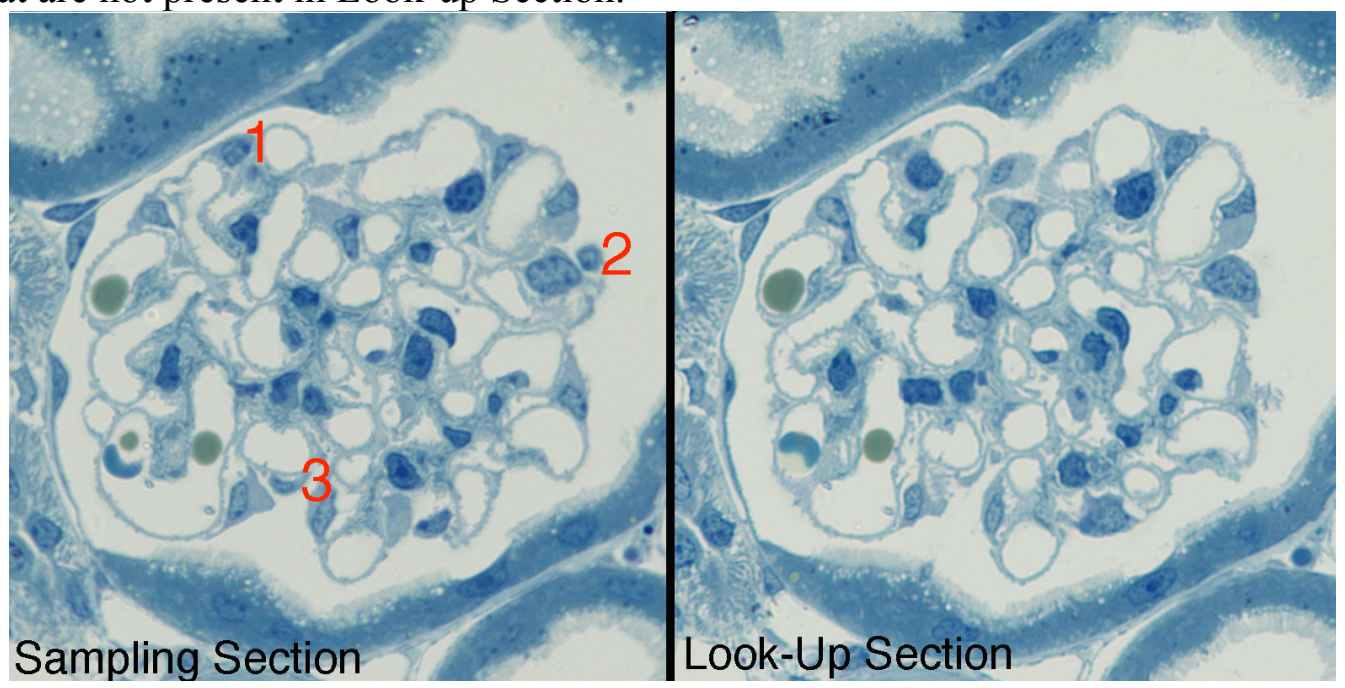

